\title{
Management of accidental extubation in patients in the prone position
}

\author{
Bosch Duran L., Pacreu Terradas S., Pardo Bedia A., Castelltort Mascó L., Sadurní \\ Sardà M., Gallart Gallego L. \\ Parc de Salut Mar, Dept of Anaesthesiology, Barcelona, Spain
}

Background: Accidental extubation in surgical prone position is an exceptional and life-threatening complication ${ }^{1}$. We present a case report and we propose a management algorithm for this accident.

Case report: A 78-year-old man, ASA III, underwent C1-C4 fixation. Airway evaluation was Mallampati Class I, mandibular subluxation $0^{\circ}$, correct thyromental distance and slight limitation of head extension. History of previous easy tracheal intubation. General anesthesia and tracheal intubation were practiced. The patient was placed in prone position and head was fixed with Mayfield ${ }^{\circledR}$ skull clamp. Anesthesia was maintained without neuromuscular blockade due to intraoperative neurophysiological monitoring. After 3 hours of surgery, accidental extubation was confirmed. We warned the surgeon and made an attempt of fiberoptic intubation in prone position, without success. A LMA Fastrach ${ }^{\circledR}$ in prone position was placed. Tracheal intubation through LMA was achieved with adequate ventilation. In this context, the patient presented oxygen desaturation and extreme bradycardia, ending up with cardiac arrest due to asystolia. We rotate the patient, started cardiopulmonary resuscitation maneuvers and administered intravenous adrenaline. Return of spontaneous circulation was achieved in 3 minutes. Surgical procedure was finished and patient had a satisfactory evolution without sequels.
Discussion: Accidental extubation must be prevented, identifying and correcting the causes. In our case, extubation was caused by perioral muscular activity, induced by intraoperative neurophysiological monitoring, and copious secretions. These factors decreased the adhesion of the paper tape used to attach the tracheal tube. We have changed the model of tape and modified tube attachment standards. If no formal contraindication exists, atropine is administered. We decided to design an algorithm (Figure 1 ) $^{2}$ that defines the team roles. The strategy is based on the ability of adequate prone mask ventilation, the ability of LMA ventilation and the difficulty of tracheal intubation. A previous simulation of the scene could be useful, assigning a task to every person involved. The path of the algorithm has to be previously discussed and decided, in order to act fast and effectively.

\section{References:}

1. Spond M, Burns $T$, Rosenbaum $T$, et al. Crisis management of accidental extubation in a prone-positioned patient with Klippel-Feil Syndrome. A \& A Case Reports. 2016; 6:383386.

2. Hung $\mathrm{MH}$, Fan SZ, Lin $\mathrm{CP}$, et al. Emergency airway management with fiberoptic intubation in the prone position with a fixed flexed neck. Anesth Analg 2008; 107:1704-1706.

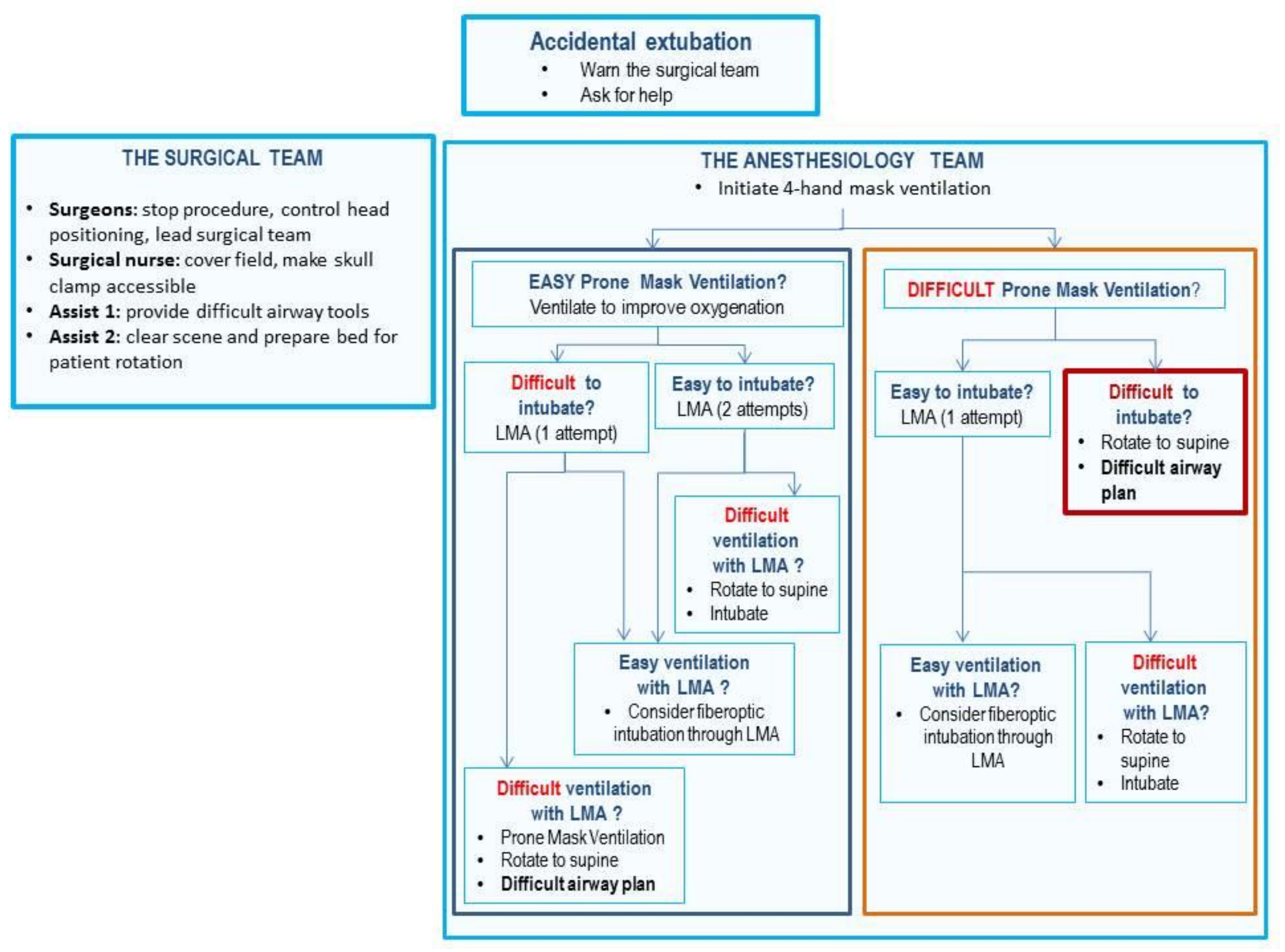

Learning points:

- We believe that insertion of the LMA Fastrach $\AA^{\circledR}$ should be a good choice.

- It would be the first option in patients in prone position. 